\title{
Micellization Behavior of Surface Active Ionic Liquids Having Aromatic Counterions in Aqueous Media
}

\author{
Gurbir Singh, Gagandeep Singh, Tejwant Singh Kang* \\ Department of Chemistry, UGC-centre for Advance Studies - I, Guru Nanak Dev University, \\ Amritsar, 143005, India
}

\section{Supporting Information}

*To whom correspondence should be addressed:

e-mail: tejwantsinghkang@gmail.com; tejwant.tej@gmail.com Tel: +91-183-2258802-Ext-3207 


\section{Annexure S1.}

\section{Synthesis and characterization of $\left[\mathrm{C}_{16} \mathrm{mim}\right][\mathrm{X}]$ ILs:}

Equal molar amount of $\left[\mathrm{C}_{16} \mathrm{mim}\right][\mathrm{Cl}]$ and the corresponding organic sodium sulfonate salt were mixed in butanol and stirred at $25{ }^{\circ} \mathrm{C}$ overnight. Following that, the reaction mixture was filtered to remove $\mathrm{NaCl}$, formed during the reaction. The butanol was removed by rotary evaporation. The residue was washed with diethylether to remove the residual butanol, if any. The obtained ILs were dried 24 hours under vacuum. The molecular structures of the synthesized ILs were confirmed by ${ }^{1} \mathrm{H}$ NMR and mass spectrometry. ${ }^{1} \mathrm{H}$ NMR spectra were recorded on Brüker Ascend 500 spectrometer (AVANCE III HD console) in $10 \% \mathrm{D}_{2} \mathrm{O}$ and $90 \% \mathrm{H}_{2} \mathrm{O}$ mixture as solvent mixture. High resolution mass spectra were recorded on Waters Q-ToF micromass equipment using ESI as the ion source.

\section{${ }^{1}$ H NMR and mass spectroscopy data for synthesized ILs:}

[C $\left.\mathbf{C}_{\mathbf{1 6}} \mathbf{m i m}\right][\mathbf{H B S}]:{ }^{1} \mathrm{H} \mathrm{NMR}\left(500 \mathrm{MHz}, 10 \% \mathrm{D}_{2} \mathrm{O}\right.$ and $90 \% \mathrm{H}_{2} \mathrm{O}, \delta$-ppm) $0.88\left(\mathrm{t}, 3 \mathrm{H}, \mathrm{CH}_{3}\right), 1.29$ (

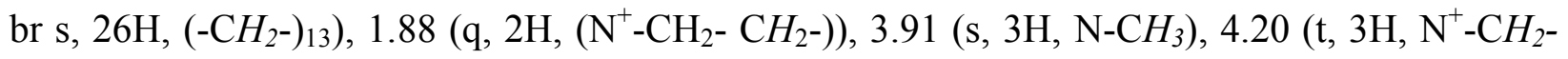

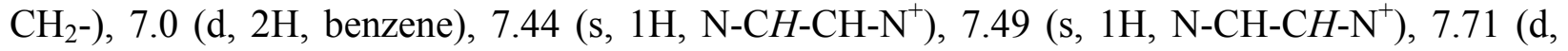
$2 \mathrm{H}$, benzene), $8.72\left(\mathrm{~s}, 1 \mathrm{H}, \mathrm{N}-\mathrm{CH}-\mathrm{N}^{+}\right.$). ESI-HRMS positive ions $\mathrm{m} / \mathrm{z}\left(\right.$ for $\left.\mathrm{C}_{20} \mathrm{H}_{39} \mathrm{~N}_{2}^{+}\right)$: 307.3171 , 308.3107 and negative ions $\mathrm{m} / \mathrm{z}$ (for $\mathrm{C}_{6} \mathrm{H}_{5} \mathrm{O}_{4} \mathrm{~S}^{-}$): $173.0108,174.0140$

[C $\left.\mathbf{C}_{\mathbf{1 6}} \mathbf{m i m}\right][\mathrm{BS}]:{ }^{1} \mathrm{H}$ NMR $\left(500 \mathrm{MHz}, 10 \% \mathrm{D}_{2} \mathrm{O}\right.$ and $\left.90 \% \mathrm{H}_{2} \mathrm{O}, \delta-\mathrm{ppm}\right) 0.88\left(\mathrm{t}, 3 \mathrm{H}, \mathrm{CH}_{3}\right), 1.29$ ( br s, 26H, $\left.\left(-\mathrm{CH}_{2^{-}}\right)_{13}\right), 1.88\left(\mathrm{q}, 2 \mathrm{H}, \mathrm{N}^{+}-\mathrm{CH}_{2^{-}} \mathrm{CH}_{2^{-}}\right), 3.91\left(\mathrm{~s}, 3 \mathrm{H}, \mathrm{N}-\mathrm{CH}_{3}\right), 4.20\left(\mathrm{t}, 3 \mathrm{H}, \mathrm{N}^{+}-\mathrm{CH}_{2^{-}} \mathrm{CH}_{2^{-}}\right.$ ), $7.44\left(\mathrm{~s}, 1 \mathrm{H}, \mathrm{N}-\mathrm{CH}-\mathrm{CH}-\mathrm{N}^{+}\right), 7.49$ (s, 1H, N-CH-CH-N $\left.{ }^{+}\right), 7.57$ (d, 2H, benzene), 7.61(t, 1H, benzene), $7.84\left(\mathrm{~d}, 2 \mathrm{H}\right.$, benzene), $8.72\left(\mathrm{~s}, 1 \mathrm{H}, \mathrm{N}-\mathrm{CH}-\mathrm{N}^{+}\right)$. ESI-HRMS positive ions $\mathrm{m} / \mathrm{z}$ (for $\mathrm{C}_{20} \mathrm{H}_{39} \mathrm{~N}_{2}^{+}$):307.3171, 308.3107 and negative ions $\mathrm{m} / \mathrm{z}$ (for $\mathrm{C}_{6} \mathrm{H}_{4} \mathrm{O}_{3} \mathrm{~S}^{-}$):156.9924, 157.0033, 157.0163

[C $\left.\mathbf{1 6}_{\mathbf{1 6}} \mathbf{m i m}\right][\mathrm{PTS}]:{ }^{1} \mathrm{H}$ NMR (500 MHz, 10\% $\mathrm{D}_{2} \mathrm{O}$ and 90\% $\left.\mathrm{H}_{2} \mathrm{O}, \delta-\mathrm{ppm}\right)$ 0.869-0.898 (t, 3H, $\left.\mathrm{CH}_{3}\right), 1.29$ ( br s, 26H, $\left.\left(-\mathrm{CH}_{2}\right)_{13}\right), 1.88$ (q, 2H, (q, 2H, N $\left.{ }^{+}-\mathrm{CH}_{2}-\mathrm{CH}_{2^{-}}\right), 2.43$ (s, 3H, $\left.\mathrm{CH}_{3}\right), 3.91$ (s, 3H, N-CH3), 4.20 (t, 3H, N $\left.-\mathrm{CH}_{2-} \mathrm{CH}_{2-}\right), 7.40$ (d, 2H, benzene), 7.44 (s, 1H, N-CH-CH-N ${ }^{+}$), $7.49\left(\mathrm{~s}, 1 \mathrm{H}, \mathrm{N}-\mathrm{CH}-\mathrm{CH}-\mathrm{N}^{+}\right), 7.72\left(\mathrm{~d}, 2 \mathrm{H}\right.$, benzene), 8.72(s, 1H, N-CH-N $\left.{ }^{+}\right)$. ESI-HRMS positive ions $\mathrm{m} / \mathrm{z}$ (for $\mathrm{C}_{20} \mathrm{H}_{39} \mathrm{~N}_{2}{ }^{+}$): $307.3171,308.3107$ and negative ions $\mathrm{m} / \mathrm{z}\left(\right.$ for $\mathrm{C}_{7} \mathrm{H}_{7} \mathrm{O}_{3} \mathrm{~S}^{-}$): 171.0299, 172.0332 . 

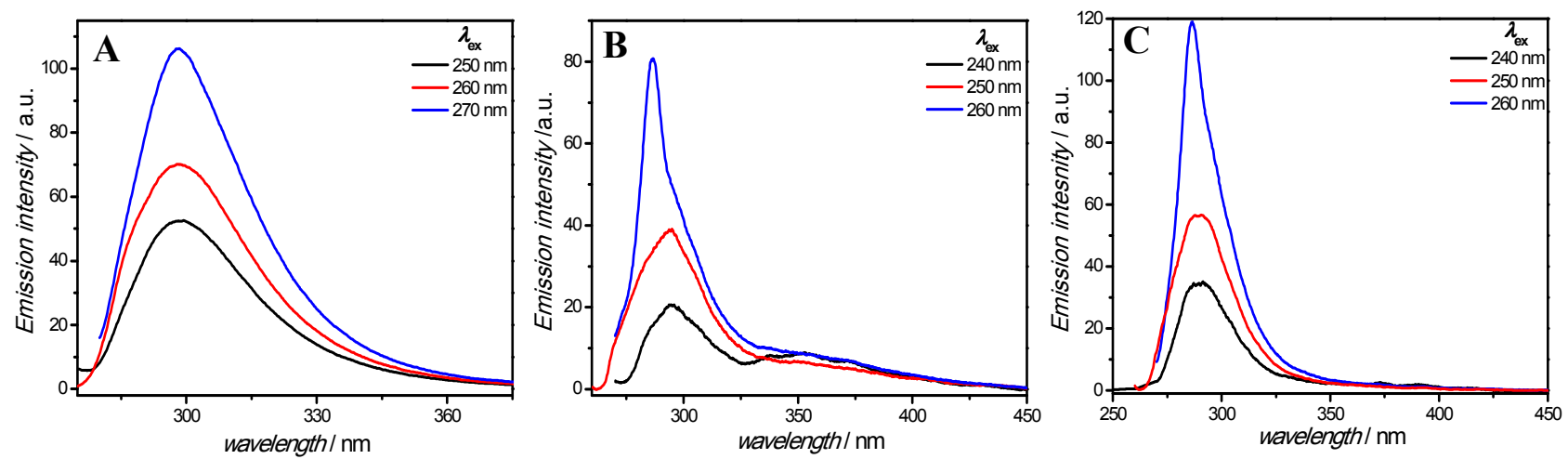

Figure S1: Emission spectra of aromatic anions of different ILs at varying excitation wavelengths $\left(\lambda_{\text {ex }}\right)$ as $(\mathrm{A})\left[\mathrm{C}_{16} \mathrm{mim}\right][\mathrm{HBS}]$; $(\mathrm{B})\left[\mathrm{C}_{16} \mathrm{mim}\right][\mathrm{BS}]$; and $(\mathrm{C})\left[\mathrm{C}_{16} \mathrm{mim}\right][\mathrm{PTS}]$ at $298.15 \mathrm{~K}$.
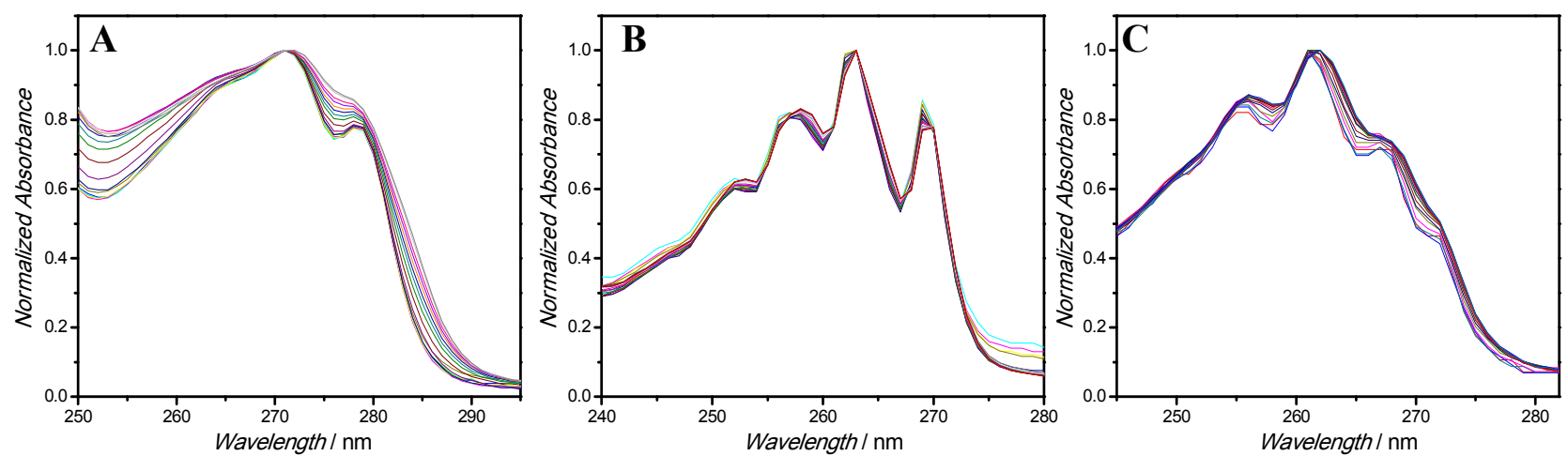

Figure S2: Normalized UV-Vis absorption spectra of different ILs corresponding to aromatic anions of in their aqueous solutions at different concentrations ranging from below cmc to above cmc as (A) [C $\left.{ }_{16} \mathrm{mim}\right][\mathrm{HBS}]$; (B) $\left[\mathrm{C}_{16} \mathrm{mim}\right][\mathrm{BS}]$; and (C) $\left[\mathrm{C}_{16} \mathrm{mim}\right][\mathrm{PTS}]$ at $298.15 \mathrm{~K}$. 

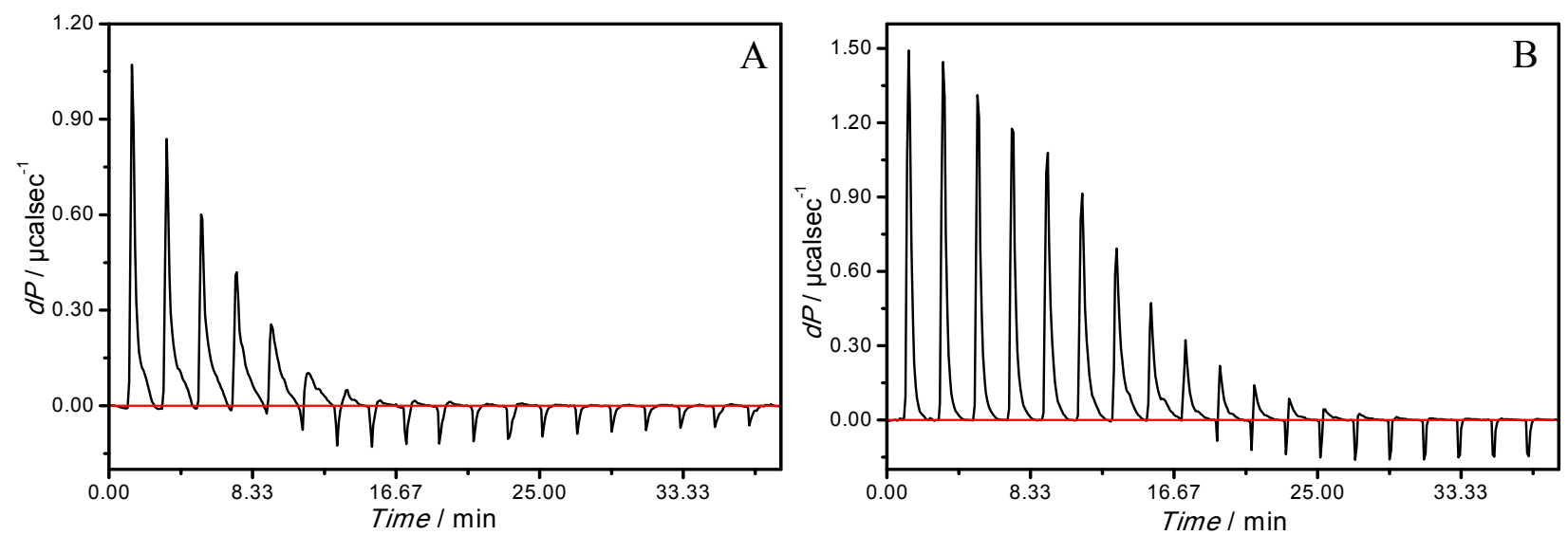

Figure S3: Differential power profile obtained for aqueous solutions of $(\mathrm{A})\left[\mathrm{C}_{16} \mathrm{mim}\right][\mathrm{BS}]$ and (B) $\left[\mathrm{C}_{16} \mathrm{mim}\right][\mathrm{PTS}]$ at $298.15 \mathrm{~K}$.

Table S1. The magnitude of change in chemical shift values $\left(\Delta \delta_{\mathrm{ppm}}\right)$ for different protons as a consequence of micellization of ILs at 298.15 K.

\begin{tabular}{|c|c|c|c|c|c|c|c|c|c|c|c|c|}
\hline \multicolumn{13}{|c|}{ Change in chemical shift values $/ \Delta \delta_{\mathrm{ppm}}$} \\
\hline & $\mathrm{H}_{\mathrm{a}}$ & $\mathrm{H}_{\mathrm{b}}$ & $H_{c}$ & $\mathrm{p}-\mathrm{CH} 3$ & $\mathrm{H}_{\mathrm{h}}$ & $\mathrm{H}_{\mathrm{d}}$ & $\mathrm{H}_{4}$ & $\begin{array}{c}\mathrm{H}_{3} \& \\
\mathrm{H}_{5}\end{array}$ & $\begin{array}{l}\mathrm{H}_{2} \& \\
\mathrm{H}_{6}\end{array}$ & $\mathrm{H}_{\mathrm{g}}$ & $\mathrm{H}_{\mathrm{f}}$ & $\mathrm{H}_{\mathrm{e}}$ \\
\hline$\left[\mathrm{C}_{16} \mathrm{mim}\right][\mathrm{HBS}]$ & 0.018 & |- & 0.19 & - & -0.08 & -0.20 & - & -0.110 & -0.079 & -0.167 & -0.100 & -0.063 \\
\hline$\left[\mathrm{C}_{16} \mathrm{mim}\right][\mathrm{BS}]$ & 0.028 & & 0.229 & & -0.044 & -0.166 & -0.188 & -0.149 & -0.033 & -0.065 & -0.072 & \begin{tabular}{|l|}
0.041 \\
\end{tabular} \\
\hline$\left[\mathrm{C}_{16} \mathrm{mim}\right][\mathrm{PTS}]$ & 0.031 & - & 0.226 & -0.102 & -0.046 & -0.20 & - & -0.176 & -0.052 & -0.062 & -0.065 & 0.035 \\
\hline
\end{tabular}

